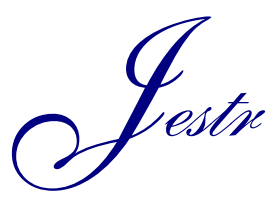

\title{
Image Fusion Methods: A Survey
}

\section{Saleha Masood ${ }^{1}$, Muhammad Sharif ${ }^{1}$, Mussarat $\operatorname{Yasmin}^{1}$,Muhammad Alyas Shahid ${ }^{1, *}$ and Amjad $\operatorname{Rehman}^{2}$}

${ }^{1}$ Department of Computer Science, Comsats Institute of Information technology WahCantt

${ }^{2}$ MIS Department CBA Salman bin Abdulaziz University Alkharj KSA

Received 17 October 2016; Accepted 13 May 2017

\begin{abstract}
Image fusion is an approach which is used to amalgamate the corresponding features in a sequence of input images to a single composite image that preserves all the significant features of the input images. Image fusion is also known as pansharpening. It is a method which is used to integrate and add the geometric detail of a high-resolution panchromatic (Pan) image and the information of color of a low-resolution multispectral (MS) image for the production of a high-resolution MS image. This methodology is mainly most important and significant for any large-scale applications. Image fusion classification based on its systems (models and algorithms) are considered and overviewed in this survey. The basic two algorithms categories are analyzed and compared to each other, in the analysis and discussion section the major points to be considered while performing image fusion are highlighted.
\end{abstract}

Keywords: Image fusion methods, Feature matching, Image resampling, Spatial Domain Fusion, Temporal Domain Fusion

\section{Introduction}

The perception of fusing a sequence and series of images into a single image started in the past around the era comprising 1950's and 1960's, by means of the exploration on behalf of useful approaches of amalgamation pictures commencing numerous devices with the most common device called sensor to deliver a compound picture might be utilized to well classify ordinary and synthetic entities [1]. Expressions likeamalgamation, permutation, collaboration, incorporation, and numerous additional concepts that direct further or fewer the equivalent notions have since seemed in the fiction [2]. In the application of remote sensing communal, the succeedingdescription has been embraced:

"A formal framework in which means and tools for expressing the alliance of data are originating from different sources called Data fusion. It aims to obtain information of greater and better quality; the exact definition of 'greater quality' will depend upon the application" [3].

Image fusion, in general, a procedure through which two or extra imageries or pictures are joined into a solo image holding the significant structures and topographies from each of the original images [4]. The fusion of images is frequently essential for pictures attained through diverse mechanism modalities or acquired methods of the identical prospect or entities [5]. Significant solicitations of image fusion comprise medicinal processing of images for better diagnostic and interpretation of diseases, imaging in the context of analyzing microscopic details, remote sensing, engineering, computer sciences including computer vision and robotics. Fusion methodscomprise the modest technique of averaging pixels within the imageand moving towards furthercomplex and problematic approaches like PCA and imagefusion using the method of wavelet transforms in the process of image fusion [6]. Numerous image fusion methods might be renowned, contingent on whether the pictures are blended and mixed in the domain of 3dimensional area or they are distorted into an alternativearea, and their alters fused [7].

\subsection{The aim of the image fusion}

The basic goals of performing image fusion method can be analyzed in the diagram of figure 1 .

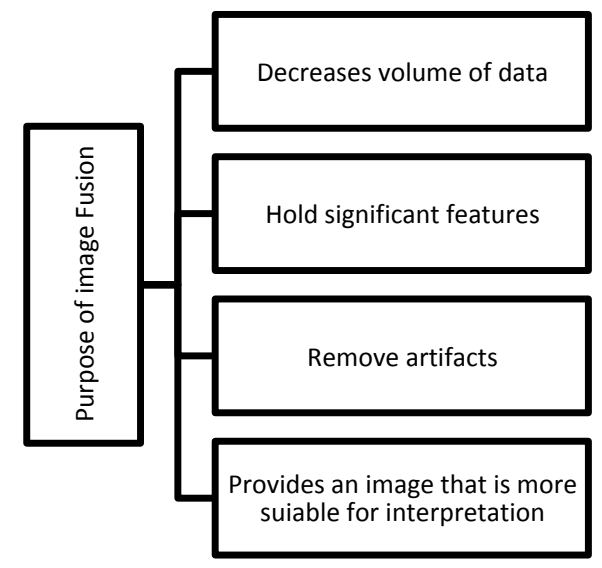

Fig 1. Aim of image fusion

\section{Image Fusion Systems}

There are two main kinds of image fusion systems, which are shown in figure 2. 


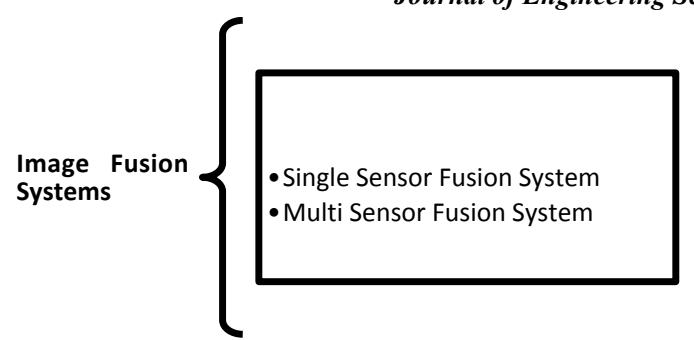

Fig 2. Image Fusion Systems

\subsection{Single Sensor Fusion System (SSFS)}

In the system where there is only one sensor, images are taken and captured using a single sensor, images are taken in a sequence of a particular scene and then the sequence of images is fused into a single image[8]. There is a major drawback of using a single sensor as it is sensor capability dependent so the problems can arise using a single sensor include in [9].

The first and most significant drawback is the capability of the sensor as they are designed only for some specific conditions and scenarios so the capability problem introduces further issues including resolution, circumstances under which it can work and the dynamic range under which the sensor can work[10].

\subsection{Multi-Sensor Fusion System (MSFS)}

In the case of a MSFS, numerous sensors are utilized for the purpose of taking and capturing images [11]. A sequence of images is captured with different sensors which are then fused into a single image that presents all in focus image. MSFS of images was basically introduced to overcome the drawbacks of single sensor fusion system and thus offers a number of benefits over single sensor image fusion system [12]. These benefits include:

\subsubsection{Benefits:}

The welfares of MSFS of images are comprised of:

i. As when we were talking about multiple sensors we said that different sensors operate under different conditions and thus providing a capability of operating under various conditions, as a result, increasing the range of conditions under which the system can operate and thus provides more effective and efficient image fusion system [1314].

ii. Different sensors have a diverse range of temporal and spatial characteristics so combining information from various sensors will result in increased range of temporal and spatial characteristics within the fused image [15].

iii. Important information and features from multiple images taken from multiple sensors are fused into a single image so the probability of uncertainty and error in the resultant image is far less as compared to the image fused using a single sensor image fusion system [16].

iv. There will be are duction in noise factor in the fused image and the reliability factor will increase providing a high-quality image [17].

v. The multi-sensor fusion system provides redundancy in numerous quantities that can support in structures strength [18]. In a circumstance when one or additional sensors stop working or the concert of a specific sensor fails, the method can rely on the additional sensors within the system [19].

vi. A dense and more appropriate representation of information and important features is possible through the use of multiple sensors as all the important features are stored in a single imageinstead of storing each feature separately [2021].

\section{Why Image Fusion?}

Multi-sensor statisticsblend has converted into a criticism which stresses further all-purpose standard clarifications to a quantity of solicitation circumstances [22]. Numerous conditions in image dispensation need both great threedimensional and spectral evidence in a sole image [23-24]. It is significant in the domain of remote sensing [25]. Conversely, the tools are not proficient of offering such info either throughstrategy or for the reason of analysis restrictions [26]. One conceivable answer on behalf ofthis problem is the fusion of images [27].

The process of fusing images allows the combination of diverse statistics foundations [28]. The merged image might have corresponding three-dimensional and spectral perseverance features [29]. On the other hand, the ordinary methods of image fusion can alter the spectral facts of the multispectral data while incorporationof images [30].

\section{Literature Review}

If we analyze the domain of image fusion we can see that huge work has been done in this context ranging from the spatial domain to temporal domain in the image fusion prospect. Starting from the one of the most common application of image fusion i.e. remote sensing [31] the approach basically makes use of multiple sensors to detect the error in the cotton plants. Image fusion using the concept of pixels and wavelet is presented in [32].Details of image fusion applications in the domain of remote sensing are presented in [33]. Another major application of image fusion is in the medical imaging. The fusion of MR images through the use of long and short axis is presented in [34].Similarly, the fusion of liver images can be analyzed in [35]. Another major application where image fusion is more applicable and is applied is the area of image enhancement; pattern selective color fusion is an example in this regard [36]. In the case of remote sensing the concept of multispatial and spectral details of images is really important [37]. A wavelet-based image focus fusion can be analyzed in [38]. Comparative image fusion is presented in [39]. Contour based transform is another category of image fusion [40]. Image fusion using the concept of DWT is the most widely and advantageous approach as it offers a number of advantages in the context of fusing images [41]. DWT with the combination of the multi-focus concept is presented in [42]. The concept of Multispectral and Panchromatic in the image fusion process can be analyzed in [43]. Fuzzy logic is another branch of image fusion [44]. The application of image fusion in the transportation system is presented in [45]. The analysis of image fusion algorithm being used is another important concern in the prospect of image fusion [46]. Feature fusion is also performed in the image fusion category as the corresponding features in the sequence of images into a solo composite image are the basic goal of 
image fusion [47]. The combination of wavelet and fuzzy logic concept for image fusion is presented in [48]. A novel approach by taking into account the concept of multi-focus for image fusion can be analyzed in [49]. Using curvelet transforms for the image fusion is an important method in the process of fusing images [50]. Evaluating the spatial and spectral details of images after applying an image fusion technique is an important step[51]. Guided filtration process for image fusion is presented in [52].Multimodal and multitemporal image fusion method can be analyzed in [53]. ICA independent component analysis approach can also be utilized for performing a fusion of sequence of images [54]. Quantitative analysis of image fusion algorithm is another major area of concern [55]. The application of image fusion in the context of signal processing is presented in [56]. Region segmentation and spatial frequency can be the two important factors in the process of multi-focus image fusion [57]. The fusion of region and wavelet-based image fusion is presented in [58].

\section{Image fusion Models}

There are three types of image fusion models, which are given in figure 3 .
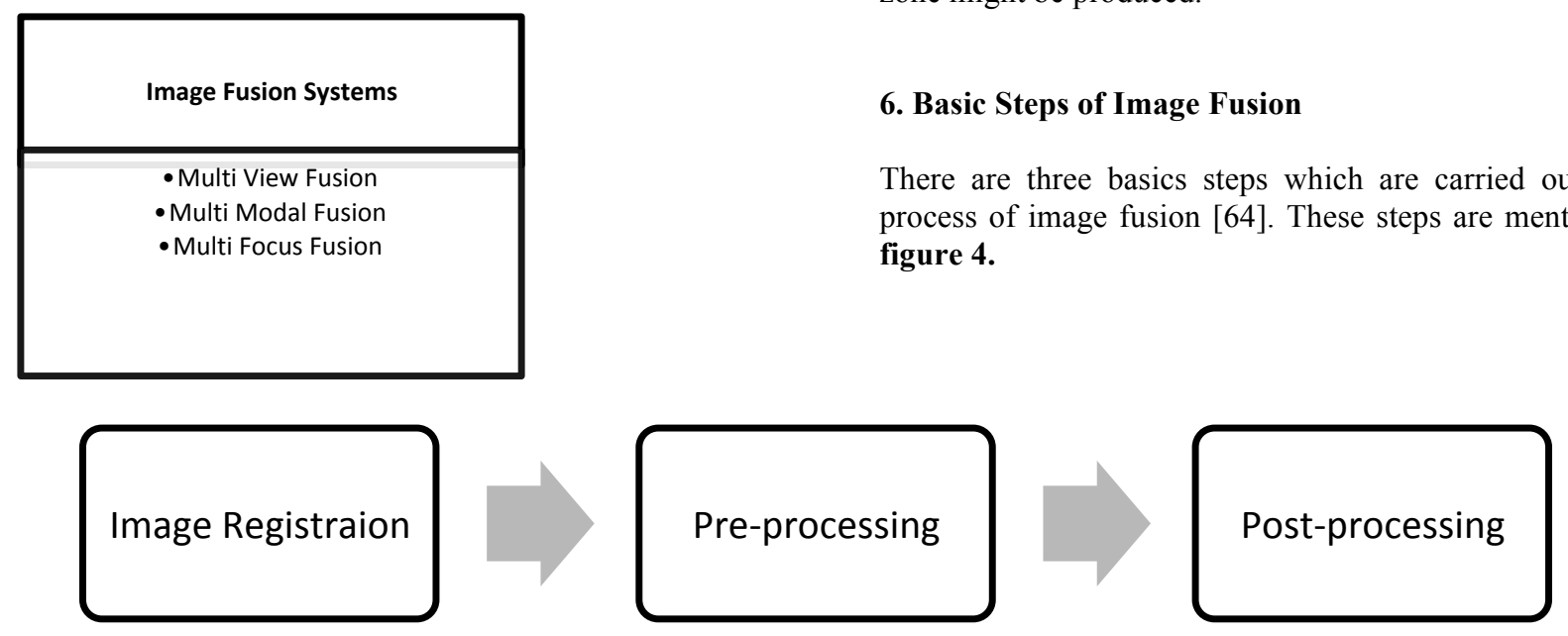

Fig 4. Image Fusion Basic Steps

\subsection{Image Registration}

The process of image registration aligns the sequence of images in order to overlap the corresponding features and details correctly [65].

\subsection{Pre-Processing:}

In the procedure of image fusion, the basic aim of this step is to make the images more suitable for the algorithm of image fusion. [66-67]

\subsection{Post-Processing:}

This step is basically dependent on the kind or category of the display being used in the fusion process. Another dependability of this process involves the inclination of a human operator. [68]

\subsection{Image Registration:}

The image registration is the main and basic step performed in the image fusion system [69].The process of image registration in image fusion works on the fact that the
Fig 3. Image Fusion Models

\subsection{Multi-View Fusions}

The multi-view approach of image fusion has the characteristic to mergenumerous $3 \mathrm{D}$ data of the equivalent importance and matching samples by commencing diverse viewpoints within a single resultant image.

\subsection{Multi-Modal Fusions}

In the case of multimodal image fusion various models are combined and fused into a single image [59].

\subsection{Multi Focus Fusions}

The approach of multi-focus fusion of images merge and blend the important details and features from two or more images into a single image resulting in an image that is all in focus image[60]. It means that every feature or object in the output image has a proper focus [61-62].

In a situation where one site encompasses substances in diverseexpanse, the camera might be dedicated to every piece of information within the image one afterward the further, generating a group of images [63]. Then, by means ofimage fusion, a picture with superioremphasisover entire zone might be produced.

\section{Basic Steps of Image Fusion}

There are three basics steps which are carried out in the process of image fusion [64]. These steps are mentioned in figure 4. conditions under which a sensor operate, its resolution, the distortion of lens or the rate of frames can never be anticipated to counterpart with the other so it follows the principle to match the pixels among the images to map the similar features in the resultant fused image [70].

The general and most utilized method used for this purpose encompasses rotation or the geometric transformation [71]. Other methods that are also applicable involve affine, projective and polynomial methods but they are considered as progressive global approaches [72].Straightforward geometric translation or rotation is the simplest technique. Image registration is performed indifferentstepswhich are mentioned in figure 5 .

\subsubsection{Feature detection}

The Process basically detects the corresponding similar features within the images which are to be fused in a single image.

\subsubsection{Feature matching}

Here in this step, the similar corresponding features detected in the sequence of images are matched to each other. The 
step is performed by finding the correspondence between the detected features.

\subsubsection{Transform model estimation}

The mapping function is used to perform the transformation step so here in this step the parameter and the types of the estimation model to be used are figured out.

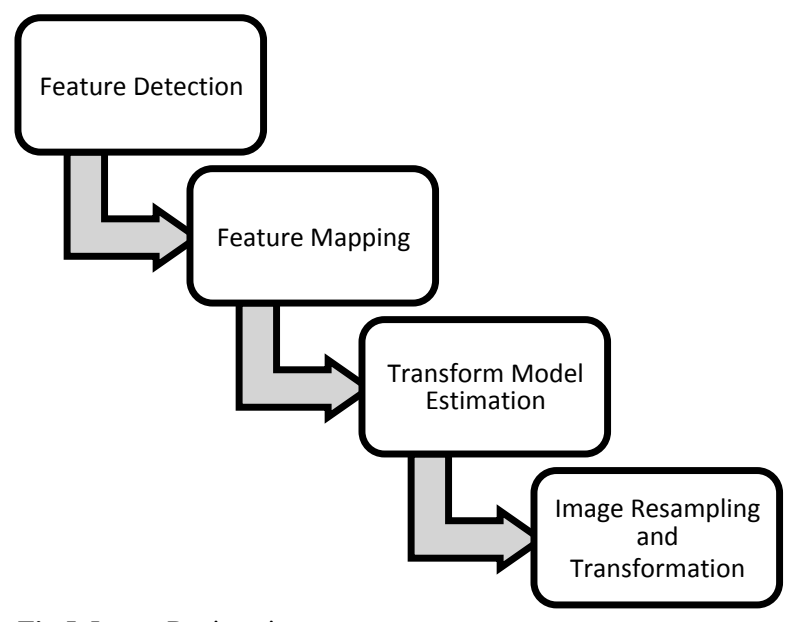

Fig 5. Image Registration steps

\subsubsection{Image resampling and transformation}

The image which is sensed in the previous step is basically transformed in this step [73-74].

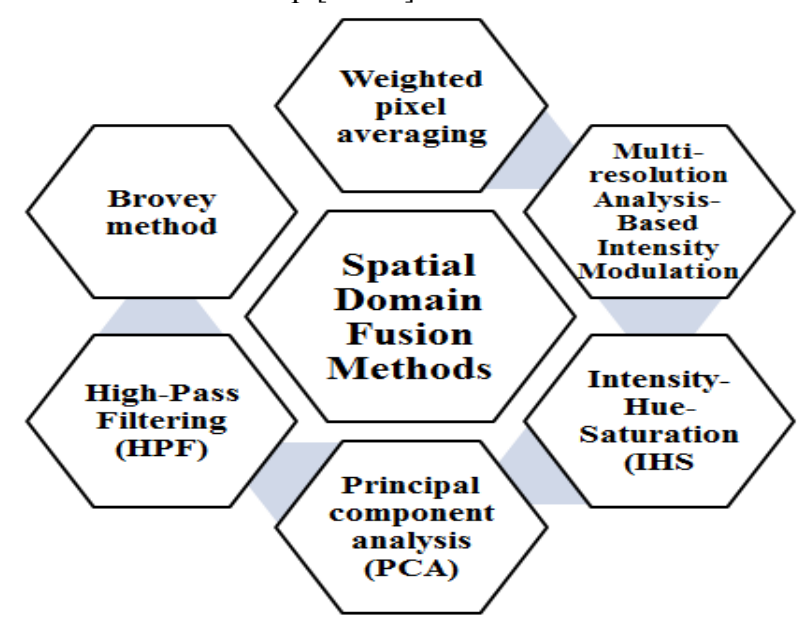

Fig 6. Spatial Domain Fusion Methods

\section{Image Fusion Classification}

Image fusion methods can be divided into two major domains which include [75]:

- Spatial Domain Fusion

- Temporal Domain Fusion

\subsection{Spatial Domain Fusion Methods:}

A Spatial Domain Fusion Method is shown in figure 6.

Here is a brief overview of each method under the category of spatial domain fusion methods.

\subsubsection{Weighted pixel averaging:}

Weighted pixel averaging approach under the spatial domain of the image fusion is the simplest method in this prospect. The basic formula of performing this operation is:
$\mathbf{F}(\mathbf{x}, \mathbf{y})=\mathbf{W}_{\mathrm{A}} * \mathbf{A}(\mathbf{x}, \mathbf{y})+\mathbf{W}_{\mathbf{B}} * \mathbf{B}(\mathbf{x}, \mathbf{y})$

Where in the above equation $\mathrm{W}_{\mathrm{A}}, \mathrm{W}_{\mathrm{B}}$ variables are scalars

The basic advantage offered by this approach is that it has the potential to lessen or decrease the present noise or artifacts within the source of image [76]. But while decreasing the noise present in the image it also destroys the significant features of the image [77].

The resultant image by the process is a low contrast image that gives a washed-out look.

\subsubsection{Brovey Transformations}

The brovy transformations carry out the task of image fusion by taking into account the factor of chromaticity transform [78-80].Applications where images are to be fused into a single composite image by taking the sequence of images through diverse sensors then brovy transformations are considered to be the simplest and most applicable approach. As in such a case, there exists limitation of three bands so the aim of BT is to standardize the various spectral i.e. 3dimensional bands for the display of RGB. Another reason why BT is the most applicable method is such a condition is that.

They add the component of brightness and intensity in the resultant fused image by performing multiplication of the result with the anticipated data [81].

\subsubsection{High-Pass Filtering (HPF)}

HRPI and LRMIs are the two major factors in this regard as they are combined to acquire the factor of HRMIs [8284],performing this task is the basic and vital aim of high pass filtering in the image fusion process.

The basic workflow of HRF is comprised of acquiring the information with high frequency. This information is projected by performing the process of filtration where high pass filter is used to filter the HRPI. The similar task can be performed by subtracting the LRPI from the native HRPI. The approach offers the advantage that 3 -dimensional details present in the image are preserved. The reason is that as it holds the high-frequency details of the images so the details of spectral and spatial domains are connected or contained within the high and low-frequency information [85].

\subsubsection{Principal component analysis (PCA)}

PCA, in general, a mathematical approach that makes use of transformations based on an orthogonal factor. The orthogonal feature results in a group of linear values that are acquired by converting a group of opinions from conceivably connected variables. The linear values being acquired are also called the principal components. The proportion of these principal components should be that they are supposed to be equal or less than the variables which are original within the images. In the prospect of multivariate analyses PCA is the easiest and simplest form [86-87].

\subsubsection{Intensity-Hue-Saturation (IHS)}

The IHS method under the spatial domain of image fusion makes use of traditional transformations comprised of three orders which are basically extended to a new domain or arbitrary order [88]. The advantage offered by this approach is that the computation of composite image in image fusion is a fast mechanism. Together with this advantage it also has the ability to combine a massive collection of data quickly by utilizing the data from multi-spectral resampled statistics. 
But while performing this blend it distorts the original colors present within the images [88].

\subsubsection{Multi-resolution Analysis-Based Intensity} Modulation

The origins of MRAIM were introduced by Wang [90]. The basics of this approach rely on the GIF approach. The advantage offered by this approach is that a case where the ratio is present as a random integer, performing fusion using this approach will provide optimal results.

\subsection{Temporal Domain Fusion Methods}

A complete and elaborated Temporal Domain Fusion Method is shown in figure 7.

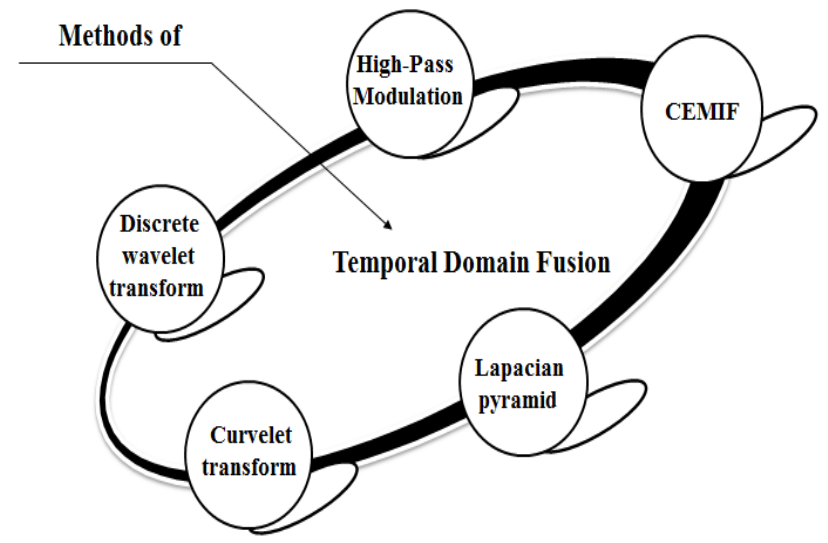

Fig 7. Temporal Domain Fusion Methods

Here is a brief overview of each method under the category of temporal domain fusion methods.

\subsubsection{High-Pass Modulation}

HPM [91] makes use of modulation coefficients in order to handover the information contained within the highfrequency domain to the LRMIs. The use of modulation coefficients in the HPM equals the proportion among the LRPI and the LRMIs.

The corresponding precise approach is expressed in [92].

\subsubsection{CEMIF}

The origins of this approach were projected by Xydeas and Petrovic. The foundation of this approach relies on the concept of multi-resolution contacting a lessen number of variables. The CEMIF is an adaptive approach [93]. The advantage offered by this approach is that it reduces the complexity and difficulty involved in the computational step within the multi-resolution approaches. The major examples under this category involve wavelet transform and Pyramid of Laplacian. It also has the ability to hold the quality of the image after performing the fusion process. The decomposition that is being kept presents an easy form of conventional Gaussian-Laplacian pyramid approach.

\subsubsection{Pyramidal Method}

The Pyramidal method under the category of the temporal domain of image fusion makes use of a data structure that is comprised of a sequence of band pass or the low pass copies of the original image. In such a case every copy presents statistics of diverse scale. The advantage offered by this approach is that it results in a high contrast, more clear and attractive and sharp image by preserving the basic and significant features of the images in a single composite image [94].

\subsubsection{Laplacian Pyramid}

When talking about the pyramid of the image we discussed that is basically comprised of copies of the band and low pass filters with each band presenting a different level and scale information of the image. Here in the case of a pyramid, the density is being decreased in steady phases. Another feature of the pyramid is that in a series of images each next image is a filtered version of its previous image. The resultant image presents the image at the lowest level with the equivalent scale of the native image. The image at the lowest level encompasses the resolution details at the highest level [95].

\subsubsection{Laplacian:}

The basic goal of laplacian method is to acquire the reduced form of the original image by making use of the filtration of the original image using the low pass filter. Similarly, the next image in the pyramid is the reduced version of its previous image and this process goes on till the last image in the sequence of images. In parallel to this process averaging the levels on after another is carried out[96]. The basic formula to perform this function is given below:

$$
g_{l}(i, j)=\sum_{m=-2}^{2} \sum_{n=-2}^{2} w(m, n) g_{l-1}(2 i+m, 2 j+n)
$$

Here in the equation, $\mathrm{N}$ denotes the number of levels within the pyramid and the dimension of Lth level is denoted through the variables.

\subsubsection{Wavelet Transform method}

The discrete sampling of wavelets is the basic function performed by the DWT. The advantage offered by this approach is its tendency to favor of handle the temporal resolution [97]. Another significant advantage offered by DWT is that holds information of location together with the details of frequency factor. The domain comprised of applications of DWT is massive enoughthat it encompasses areas including mathematics, engineering, robotics science and computer science. Another major solicitation of DWT is in the domain of signal processing.

\subsubsection{Algorithm}

The basic working of DWT method encompasses a 2 level DWT of every picture within the sequence of input images. The corresponding subbands comprised of high-frequency details are then utilized to choose the merged or blended edge details in the low pass filtration process. A window of $3 \times 3$ is utilized to calculate the mean and standard deviation in order to figure out the edge statistics. The final output is acquired by means of using the inverse of DWT on the wavelet coefficients being fused [98].

There are a number of advantages which are offered by the use of DWT. They are capable of handling diverse resolutions of images it also offers a way to acquire new coefficients from the sequence of input images. And as a result provides with a wide variety of coefficients. The resultant image that is achieved by the inverse of DWT holds the information that is combined in the fused image [99].

\subsubsection{Curvelet Transform}

Curvelettransforms [100-103]is one of the major image fusion methods under the temporal category of image fusion. 
It is basically a non-adaptive method. The aim of the method is to represent multi-scale details of the objects in the images. It is a generalized version of Fourier transform. The generalization that is being made is comprised of two additional features including spatial frequency [104-105] and the location of the objects in the images. The basic application of curvelet method is in the domain when the objects within the images contain the minutest scale length. The examples that can be considered here include text, geometrical figures and lastly the most common application area cartoons. The sparser or dense results can be acquired by using a right type of image [106].

\section{Applications}

There are a number of applications of image fusion system. These applications can be analyzed in figure 8 .

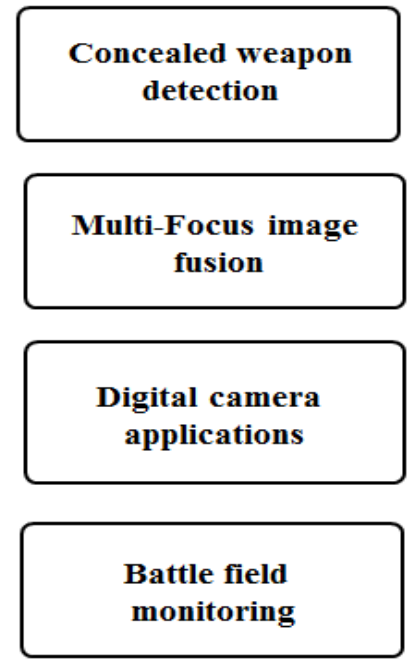

Fig 8. Image Fusion Applications

\section{Analyses and Discussion}

The overview of the image fusion approaches in the spatial and temporal domainsadduces a number of issues. This method / approachshould be considered while performing the image fusion process. Each method has its own strengths and weaknesses. The basic points are analyzed below:

With the passage of time, the requirement to merge the effective and significant features from multiple image sources increased as using a single sensor for capturing a sequence of images resulted with a number of limitations and drawbacks. Image fusion process works by representing information that is represented at different levels within a single image. A more effective move that can be taken in this regard is to categorize the different level of information within the images into a single image. This categorization can be comprised of a level, providing info about signal than a level describing feature followed by a level of symbolic representation and so on[107].

The analysis and concentration on the concept of pixel level approach of fusion basically result in an all in one combined image of a sequence of images [108]. When it is the case that a sequence of images are presented and important features are to be combined into a single image then it is considered that the solution can be presented by a human operator but the possible answer to such operation is image fusion. The quality of image fusion process should be that it should preserve all the necessary and significant features from a sequence of images into one composite image called the fused image [109]. The process of the image should be effective enough that after combining and blending important features from several images it should not result in inconsistency and artifacts in the resultant image. The orientation and location of an entity or substance within the images are the most significant factor so while performing the process of image fusion it is supposed to be invariant to the rotation and shift factor. Another factor that creates a problem when fusing a number of images is the factor of uniformity and strength of temporal feature [110].

The factors that can create the distraction in the visual process of humans are being sensitive to stirring light spurs, time reliant disparity alterations familiarized through the process of image fusion. So in order to get results which are more consistent and uniform two factors should be handled with care. These factors include temporal stability and temporal consistency [111-113].The reason of temporal stability is that the changes occurred in the gray level while merging must be caused by the gray levels within the images, not by the process of fusing the images [114]. The sequence which is being combined and merged into a single composite image should be stable and uniform. The consistency of temporality is that changes that are being introduces in the gray levels of the images should appear in the resultant image without any change of contrast factor [115].

\section{Conclusion}

The aim of the survey was to analyze the basic process of image fusion collectively with its categorization based on its modals systems and algorithms. The analysis showed that huge work has been done in the domain of image fusion but still there is enormous room for new and innovative work in this context. The analysis also has shown that every algorithm in this context has its own strengths and weaknesses. An Analyst may observe clearly, no image fusion technique is superior over another; the selection and 
effectiveness of a particular method are dependent on its application. According to research point of view, it can be concluded that PCA based image fusion techniques result in a better-enhanced image without altering the spatial and spectral details of the image. The wavelet based approaches are suitable in the applications where original values of RGB components are to be saved. And wavelet based approaches results in less distortion in the image. Lastly,proven transformations resulted in sharper images but alter the original colors of the image by making dark areas of image darker and white areas whiter in the resultant image.

This is an Open Access article distributed under the terms of the Creative Commons Attribution License

\section{References}

[1] L. Wald,Data Fusion: Definitions and Architectures-Fusion of Images Different Spatial Resolutions. Paris, France: Les Presses de l'Ecoledes Mines, 2002. .

[2] Z. Wang and A. C. Bovik, "A universal image quality index,"IEEE Signal Process. Lett., vol. 9, no. 3, pp. 81-84, Mar. 2002.

[3] L. Bentabet, S. Jodouin, D. Ziou, and J. Vaillancourt,"Road vectors update using SAR imagery: A snake-based method,"IEEE Trans. Geosci.Remote Sens., vol. 41, no. 8, pp. 1785-1803, Aug. 2003

[4] Soo-Mok Jung, Sung-Chul Shin, HyunkiBaik and Myong-Soon Park,Fast Block Matching Algorithms for Motion Estimation, Proc. of The 6th Asia-pacific Conference on Communications(APCC2000), Pages 1308-1312, October 2000, Korea

[5] J. Cox, M. L. Miller, and A. L. McKellips. Watermarking as communications with side information. Proceedings of the

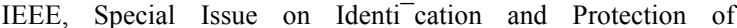
Multimedia Information, 87(7):1127\{1141,July 1999.

[6] J. J. Eggers, J. K. Su, and B. Girod. A blind watermarking scheme based on structured codebooks. In SecureImages and Image Authentication, Proc. IEE Colloquium, pages $4 / 1\{4 / 6$, London, UK, April 2000

[7] H. Wang, J. Peng and W. Wu, "Fusion algorithm for multisensor images basedondiscretemultiwavelet transform". Vision, Image and Signal Processing,Proceedings of the IEEE Vol.149, no.5:October 2002.

[8] Pajares, G., De La Cruz, JM, “ A wavelet-based image fusion tutorial”. Pattern Recognition,37,pp. 1855-1872,2004.

[9] Shutao Li, James T. Kwok, Y aonan Wang, "Combination of images with diverse focuses using the spatial frequency",Information Fusion 2(3): 169-176,2001

[10] H.H. Wang, J.X. Peng, W. Wu, "A fusion algorithm of theremote sensing image based on discrete wavelet packet", Proceedings on 2nd International Conference Machine Learning and Cybernetics, Xi'an, vol. 4, Nov. 2003, pp. 2557-2562, doi: 10.1109/ICMLC.2003.1259946

[11] E. Candes, L. Demanet, D.L.Donoho, Lexing Ying. "Fast Discrete Curvelet Transform", www.curvelet.org ,2006.

[12] L. J. Chipman, T. M. Orr, "Wavelets and Image Fusion", Proceedings of International Conference on Image Processing, Oct. 1995, vol. 3, pp. 248-251,

[13] F. Liu, J. Liu, Y. Gao, "Image Fusion based on Wedgeletand Wavelet", Proceedings of International Symposium on Intelligent Signal Processing and Communication Systems (ISPACS), Nov. 2007, pp. 682-685

[14] G. Piella. A general framework for multiresolution image fusion: from pixels to regions. Information Fusion, 4:259-280, 2003

[15] S. Gabarda, G. Cristo 'bal, Cloud covering denoising through image fusion, Image and Vision Computing 25 (5) (2007) 523530 .

[16] G. Pajares, J. Cruz, A wavelet-based image fusion tutorial, Pattern Recognition 37 (9) (2004) 1855-1872

[17] S.G. Nikolov, J.J. Lewis, R.J. O'Callaghan, D.R. Bull, C.N.Canagarajah, Hybrid fused displays: between pixel- and regionbased image fusion, in Proceedings of 7th International Conference on Information Fusion, Stockholm, Sweden, June 2004, pp. 1072-1079

[18] V. Petrovic, C. Xydeas, Sensor noise effects on signal-level image fusion performance, Information Fusion 4 (3) (2003) 167183.
[19] Luo, R. C., Lin, M. H., \&Scherp, R. S. (1988). Dynamic multisensor data fusion system for intelligent robots. Robotics and Automation, IEEE Journal of, 4(4), 386-396.

[20] Hall, D. L., \&Llinas, J. (1997). An introduction to multisensor data fusion.Proceedings of the IEEE, 85(1), 6-23.

[21] Wang, Z., Ziou, D., Armenakis, C., Li, D., \& Li, Q. (2005). A comparative analysis of image fusion methods. Geoscience and Remote Sensing, IEEE Transactions on, 43(6), 1391-1402.

[22] Qu, G., Zhang, D., \& Yan, P. (2002). The informationmeasures for theperformance of image fusion. Electronics letters, 38(7), 313-315.

[23] Javidi, B., Ferraro, P., Hong, S. H., De Nicola, S., Finizio, A., Alfieri, D., \&Pierattini, G. (2005). Three-dimensional image fusion by use of multiwavelength digital holography. Optics letters, 30(2), 144-146.

[24] Porter, B. C., Rubens, D. J., Strang, J. G., Smith, J., Totterman, S., \& Parker, K. J. (2001). Three-dimensional registration and fusion of ultrasound and MRI using major vessels as fiducial markers. Medical Imaging, IEEE Transactions on,20(4), 354359.

[25] Bretschneider, T., \& Kao, O. (2000). Image fusion in remote sensing. InProceedings of the 1st Online Symposium of Electronic Engineers (pp. 1-8).

[26] Simone, G., Farina, A., Morabito, F. C., Serpico, S. B., \&Bruzzone, L. (2002). Image fusion techniques for remote sensing applications. Information fusion, 3(1), 3-15.

[27] Krueger, M. W., Seemann, G., Rhode, K. S., Weber, F. M., Linton, N., Williams, S., ...\&Dössel, O. (2013). Fusion of local activation time maps and image data to personalize anatomical atrial models. In Functional Imaging and Modeling of the Heart (pp. 1-10). Springer Berlin Heidelberg.

[28] Huang, W., \& Jing, Z. (2007). Evaluation of focus measures in multi-focus image fusion. Pattern Recognition Letters, 28(4), 493-500.

[29] Swoger, J., Verveer, P., Greger, K., Huisken, J., \&Stelzer, E. H. (2007). Multi-view image fusion improves resolution in threedimensional microscopy. Opt Express, 15(13), 8029-8042.

[30] Amolins, K., Zhang, Y., \& Dare, P. (2007). Wavelet based image fusion techniques-An introduction, review and comparison. ISPRS Journal of Photogrammetry and Remote Sensing, 62(4), 249-263.

[31] Richards, J. A. (2013). Remote sensing digital image analysis: an introduction. Springer.

[32] McCarthy, C., Hancock, N., \&Raine, S. (2008, January). On-thego machine vision sensing of cotton plant geometric parameters: first results. InMechatronics and machine vision in practice (pp. 305-312). Springer Berlin Heidelberg.

[33] Schowengerdt, R. A. (2006). Remote sensing: models and methods for image processing. Academic press.

[34] Goshtasby, A. A., \& Turner, D. A. (1996). Fusion of short-axis and long-axis cardiac MR images. Computerized medical imaging and graphics, 20(2), 77-87.

[35] Chung, T. M., Liu, X. H., Chen, C. H., Sun, X. N., Chiu, N. T., \& Lee, J. X. (1997, December). Intermodality registration and fusion of liver images for medical diagnosis. In Intelligent Information Systems, 1997. IIS'97. Proceedings (pp. 42-46). IEEE.

[36] Bogoni, L., Hansen, M., \& Burt, P. (1999). Image enhancement using pattern-selective color image fusion. In Image Analysis and Processing, 1999. Proceedings. International Conference on ( $\mathrm{pp}$. 44-49). IEEE. 
[37] Zia, A., DeBrunner, V., Chinnaswamy, A., \&DeBrunner, L. (2002). Multi-resolution and multi-sensor data fusion for remote sensing in detecting air pollution. In Image Analysis and Interpretation, 2002. Proceedings. Fifth IEEE Southwest Symposium on (pp. 9-13). IEEE.

[38] Zhi-guo, J., Dong-bing, H., Jin, C., \& Xiao-kuan, Z. (2004, December). A wavelet based algorithm for multi-focus microimage fusion. In Image and Graphics, 2004. Proceedings. Third International Conference on (pp. 176-179). IEEE.

[39] Sadjadi, F. (2005, June). Comparative image fusion analysais. In Computer Vision and Pattern Recognition-Workshops, 2005. CVPR Workshops. IEEE Computer Society Conference on (pp. 88). IEEE.

[40] Zheng, Y. A., Zhu, C., Song, J., \& Zhao, X. (2006, August). Fusion of multi-band SAR images based on contourlet transforms. In Information Acquisition, 2006 IEEE International Conference on (pp. 420-424). IEEE.

[41] Jian, M., Dong, J., \& Zhang, Y. (2007, July). Image fusions based on wavelet transform. In Software Engineering, Artificial Intelligence, Networking, and Parallel/Distributed Computing, 2007. SNPD 2007. Eighth ACIS International Conference on (Vol. 1, pp. 713-718). IEEE.

[42] Wang, A., \& Zhao, P. (2008, May). Multi-focus Image Fusion with the Double-Density Dual-Tree DWT. In Image and Signal Processing, 2008. CISP'08. Congress on (Vol. 4, pp. 371-374). IEEE.

[43] Zhang, Y., \& He, M. (2008, May). Multispectral and panchromatic image fusion algorithm based on local spatial recovery model. In Image and Signal Processing, 2008. CISP'08. Congress on (Vol. 4, pp. 375-379). IEEE.

[44] Mengyu, Z., \&Yuliang, Y. (2008, October). A new image fusion algorithm based on fuzzy logic. In Intelligent Computation Technology and Automation (ICICTA), 2008 International Conference on (Vol. 2, pp. 83-86). IEEE.

[45] Wang, Z., Yang, Z., Su, Y., Chen, Y., Wu, J., \& Ding, X. (2008, October). Improved Image Fusion Algorithm Based on Wavelet Packet Analysis in Intelligent Transport System. In Natural Computation, 2008. ICNC'08. Fourth International Conference on (Vol. 6, pp. 358-362). IEEE.

[46] Zhao, C., Ding, Q., \& Li, J. (2008, October). The performance analysis of image fusion algorithm. In Computational Intelligence and Design, 2008. ISCID'08. International Symposium on (Vol. 2, pp. 83-86). IEEE.

[47] Zhang, P., \& Kong, X. (2009, March). Detecting image tampering using feature fusion. In Availability, Reliability and Security, 2009. ARES'09. International Conference on (pp. 335340). IEEE.

[48] Yong-Guang, M. A., \&Zhi-xin, L. (2009, May). A new arithmetic for image fusion based on fuzzy wavelet transform. In Information and Computing Science, 2009. ICIC'09. Second International Conference on (Vol. 2, pp. 365-368). IEEE.

[49] Zaveri, T., Zaveri, M., Shah, V., \& Patel, N. (2009, March). A novel region based multifocus image fusion method. In Digital Image Processing, 2009 International Conference on (pp. 50-54). IEEE.

[50] Mahyari, A. G., \&Yazdi, M. (2009, March). A novel image fusion method using curvelet transform based on linear dependency test. In Digital Image Processing, 2009 International Conference on (pp. 351-354). IEEE.

[51] Marcello, J., Medina, A., \& Eugenio, F. (2013). Evaluation of Spatial and Spectral Effectiveness of Pixel-Level Fusion Techniques.

[52] Li, Shutao, Xudong Kang, and Jianwen Hu. "Image fusion with guided filtering." Image Processing, IEEE Transactions on 22, no. 7 (2013): 2864-2875.

[53] Berger, C., Voltersen, M., Eckardt, R., Eberle, J., Heyer, T., Salepci, N., ...\&Pacifici, F. (2013). Multi-Modal and MultiTemporal Data Fusion: Outcome of the 2012 GRSS Data Fusion Contest.

[54] Mitianoudis, N., \&Stathaki, T. (2007). Pixel-based and regionbased image fusion schemes using ICA bases. Information Fusion, 8(2), 131-142.

[55] Gupta, S., Rajkumar, S., Vijayarajan, V., \&Marimuthu, K. (2013). Quantitative Analysis of various Image Fusion techniques based on various metrics using different Multimodality Medical Images. International Journal of Engineering and Technology, 5.

[56] Chen, B., Jiang, R., Kasetkasem, T., \&Varshney, P. K. (2004). Channel aware decision fusion in wireless sensor networks. Signal Processing, IEEE Transactions on, 52(12), 3454-3458.

[57] Li, S., \& Yang, B. (2008). Multifocus image fusion using region segmentation and spatial frequency. Image and Vision Computing, 26(7), 971-979.

[58] Lewis, J. J., O'callaghan, R. J., Nikolov, S. G., Bull, D. R., \&Canagarajah, C. N. (2004, June). Region-based image fusion using complex wavelets. In Seventh International Conference on Information Fusion (FUSION) (Vol. 1, pp. 555-562).

[59] Atrey, P. K., Hossain, M. A., El Saddik, A., \&Kankanhalli, M. S. (2010). Multimodal fusion for multimedia analysis: a survey. Multimedia systems, 16(6), 345-379.

[60] Huang, W., \& Jing, Z. (2007). Evaluation of focus measures in multi-focus image fusion. Pattern Recognition Letters, 28(4), 493-500.

[61] Wang, Z., Ma, Y., \&Gu, J. (2010). Multi-focus image fusion using PCNN. Pattern Recognition, 43(6), 2003-2016.

[62] Li, S., \& Yang, B. (2008). Multifocus image fusion using region segmentation and spatial frequency. Image and Vision Computing, 26(7), 971-979.

[63] Stathaki, Tania. Image fusion: algorithms and applications. Academic Press, 2011.

[64] Zitova, B., \&Flusser, J. (2003). Image registration methods: a survey. Image and vision computing, 21(11), 977-1000.

[65] Sayed, S., \&Jangale, S. (2010, February). Image registration \& image fusion. InProceedings of the International Conference and Workshop on Emerging Trends in Technology (pp. 1004-1004). ACM.

[66] Rinnan, Åsmund, Frans van den Berg, and Søren Balling Engelsen. "Review of the most common pre-processing techniques for near-infrared spectra." TrAC Trends in Analytical Chemistry 28, no. 10 (2009): 1201-1222.

[67] Teller, Seth J., and Carlo H. Séquin. "Visibility preprocessing for interactive walkthroughs." In ACM SIGGRAPH Computer Graphics, vol. 25, no. 4, pp. 61-70. ACM, 1991.

[68] Shen, Mei-Yin, and C-C. Jay Kuo. "Review of postprocessing techniques for compression artifact removal." Journal of Visual Communication and Image Representation 9, no. 1 (1998): 2-14.

[69] Maintz, J. B., \&Viergever, M. A. (1998). A survey of medical image registration.Medical image analysis, 2(1), 1-36.

[70] Treves, S. T., Mitchell, K. D., \&Habboush, I. H. (1998). Threedimensional image alignment, registration, and fusion. The quarterly journal of nuclear medicine: official publication of the Italian Association of Nuclear Medicine (AIMN)[and] the International Association of Radiopharmacology (IAR), 42(2), 83.

[71] Kooy, H. M., Van Herk, M., Barnes, P. D., Alexander III, E., Dunbar, S. F., Tarbell, N. J., ...\&Loeffler, J. S. (1994). Image fusion for stereotactic radiotherapy and radiosurgery treatment planning. International Journal of Radiation Oncology* Biology* Physics, 28(5), 1229-1234.

[72] Van den Elsen, P. A., Pol, E. J., \&Viergever, M. A. (1993). Medical image matchinga review with classification. Engineering in Medicine and Biology Magazine, IEEE, 12(1), 26-39.

[73] Dodgson, Neil. "Quadratic interpolation for image resampling." Image Processing, IEEE Transactions on 6, no. 9 (1997): 13221326.

[74] Parker, J. Anthony, Robert V. Kenyon, and Donald E. Troxel. "Comparison of interpolating methods for image resampling." Medical Imaging, IEEE Transactions on 2, no. 1 (1983): 31-39.

[75] Zhang, Y. (2004). Understanding image fusion. Photogrammetric engineering and remote sensing, 70(6), 657-661.

[76] Wang, Y. K., Hannuksela, M. M., Varsa, V., Hourunranta, A., \&Gabbouj, M. (2002). The error concealment features in the $\mathrm{H}$. $26 \mathrm{~L}$ test model. In Image Processing. 2002. Proceedings. 2002 International Conference on (Vol. 2, pp. II-729). IEEE.

[77] Garcia, F., Mirbach, B., Ottersten, B., Grandidier, F., \& Cuesta, A. (2010, September). Pixel weighted average strategy for depth sensor data fusion. InImage Processing (ICIP), 2010 17th IEEE International Conference on (pp. 2805-2808). IEEE.

[78] Nikolakopoulos, K. G. (2008). Comparison of nine fusion techniques for very high-resolution data. Photogrammetric Engineering and Remote Sensing, 74(5), 647.

[79] Zhang, N., \& Wu, Q. (2007, September). Effects of Brovey transform and wavelet transform on the information capacity of SPOT-5 imagery. InInternational Symposium on Photoelectronic Detection and Imaging: Technology and Applications 2007 (pp. 
$66230 \mathrm{~W}-66230 \mathrm{~W})$. International Society for Optics and Photonics.

[80] Mandhare, R. A., Upadhyay, P., \& Gupta, S (2013). PIXELLEVEL IMAGE FUSION USING BOVEYTRANSFORMER AND WAVELET TRANSFORM.

[81] Tu, T. M., Lee, Y. C., Chang, C. P., \& Huang, P. S. (2005). Adjustable intensity-hue-saturation and Brovey transform fusion technique for IKONOS/QuickBird imagery. Optical Engineering, 44(11), 116201-116201.

[82] Wang, Z., Ziou, D., Armenakis, C., Li, D., \& Li, Q. (2005). A comparative analysis of image fusion methods. Geoscience and Remote Sensing, IEEE Transactions on, 43(6), 1391-1402.

[83] Gangkofner, U. G., Pradhan, P. S., \& Holcomb, D. W. (2008). Optimizing the high-pass filter addition technique for image fusion. Photogrammetric Engineering \& Remote Sensing, 74(9), 1107-1118.

[84] Leung, L. W., King, B., \&Vohora, V. (2001, November). Comparision of image data fusion techniques using entropy and INI. In Paper presented at the 22nd Asian Conference on Remote Sensing (Vol. 5, p. 9).

[85] Helmy, A. K., Nasr, A. H., \& EL-TAWEEL, G. H. S. (2010). Assessment and evaluation of different data fusion techniques. International Journal of Computers, 4(4), 107-115.

[86] Yang, S., Wang, M., \& Jiao, L. (2012). Fusion of multispectral and panchromatic images based on support value transform and adaptive principal component analysis. Information Fusion, 13(3), 177-184.

[87] Kosesoy, I., Tepecik, A., Çetin, M., \&Mesut, A. (2012, April). A comparative analysis of image fusion methods. In Signal Processing and Communications Applications Conference (SIU), 2012 20th (pp. 1-4). IEEE.

[88] Afify, A. (2012). A New Intensity-Hue-Saturation Based Method for Fusing High-Resolution Satellite Images. International Journal of Geoinformatics, 8(4).

[89] Joseph, D., \&Jebaseeli, T. J. (2012). A Survey Of Fusion Of Remote Sensing Images To Avoid Spectral Distortion. International Journal of Engineering, 1(8).

[90] Matero, J., \&Shakeshaft, N. E. (2009). U.S. Patent No. 7,522,011. Washington, DC: U.S. Patent and Trademark Office.

[91] Li, Xiaoyong, Woogeun Rhee, Wen Jia, and Zhihua Wang. "A multi-bit FIR filtering technique for two-point modulators with dedicated digital high-pass modulation path." In Circuits and Systems (ISCAS), 2015 IEEE International Symposium on, pp. 894-897. IEEE, 2015.

[92] Kulkarni, J. S. (2011, April). Wavelet transform applications. In Electronics Computer Technology (ICECT), $2011 \quad 3 \mathrm{rd}$ International Conference on (Vol. 1, pp. 11-17). IEEE.

[93] Ghedini, M., Golemme, A., Aiello, I., Godbert, N., Termine, R., Crispini, A., ...\&Belviso, S. (2011). Liaisons between photoconductivity and molecular frame in organometallic Pd (ii) and Pt (ii) complexes. Journal of Materials Chemistry,21(35), 13434-13444.

[94] Simone, G., Farina, A., Morabito, F. C., Serpico, S. B., \&Bruzzone, L. (2002). Image fusion techniques for remote sensing applications. Information fusion,3(1), 3-15.

[95] Liao, C. Z., Liu, Y. S., \& Jiang, M. Y. (2013). Multifocus Image Fusion Using Laplacian Pyramid and Gabor Filters. Applied Mechanics and Materials, 373, 530-535.

[96] Lo, A. (2013). Witten Laplacian method for generalized models of Kactype.Journal of Mathematical Physics, 54, 063505.

[97] Sapkal, R. J., \&Kulkarni, S. M. (2013, April). Innovative image fusion algorithm based on fast discrete curvelet transform with different fusion rules. InInformation\& Communication Technologies (ICT), 2013 IEEE Conference on(pp. 1070-1074). IEEE.
[98] Rioul, O., \& Duhamel, P. (1992). Fast algorithms for discrete and continuous wavelet transforms. Information Theory, IEEE Transactions on, 38(2), 569-586.

[99] Bracewell, R. N., \&Bracewell, R. N. (1986). The Fourier transform and its applications (Vol. 31999). New York: McGraw-Hill.

[100] Verma, Monika, Vandana Dixit Kaushik, and Vinay Kumar Pathak. "An Efficient Deblurring Algorithm on Foggy Images using Curvelet Transforms." In Proceedings of the Third International Symposium on Women in Computing and Informatics, pp. 426-431. ACM, 2015.

[101] Arivazhagan, S., and S. Nirmala. "Rotation and Scale Invariant Texture Classification Using Gabor and Curvelet Transforms." International Journal of Tomography \& Simulation ${ }^{\mathrm{TM}} 28$, no. 2 (2015): 94-105.

[102] Ansari, Rizwan Ahmed, and Krishna Mohan Buddhiraju. "kmeans based hybrid wavelet and curvelet transform approach for denoising of remotely sensed images." Remote Sensing Letters 6, no. 12 (2015): 982-991.

[103] Bains, AmandeepKaur, and PrabhneetSandhu. "Image Denoising using Curvelet Transform." (2015).

[104] King, J. L., M. P. Lowe, and N. A. Crowder. "Contrast adaptation is spatial frequency specific in mouse primary visual cortex." Neuroscience 310 (2015): 198-205.

[105] Pérez, J. M., M. Jofre, P. Martínez, M. A. Yáñez, V. Catalan, and V. Pruneri. "An image cytometer based on angular spatial frequency processing and its validation for rapid detection and quantification of waterborne microorganisms." Analyst (2015).

[106] Choi, M., Kim, R. Y., Nam, M. R., \& Kim, H. O. (2005). Fusion of multispectral and panchromatic satellite images using the curvelet transform. Geoscience and remote sensing letters, IEEE, 2(2), 136-140.

[107] Zhang, Z., \& Blum, R. S. (2001). A hybrid image registration technique for a digital camera image fusion application. Information Fusion, 2(2), 135-149.

[108] Rockinger, O., \& Fechner, T. (1998, July). Pixel-level image fusion: the case of image sequences. In Aerospace/Defense Sensing and Controls (pp. 378-388). International Society for Optics and Photonics.

[109] Xydeas, C. S., \&Petrovic, V. (2000). Objective image fusion performance measure. Electronics Letters, 36(4), 308-309.

[110] Kim, J., \& Chen, T. (2003). Combining static and dynamic features using neural networks and edge fusion for video object extraction. IEE Proceedings-Vision, Image and Signal Processing, 150(3), 160-167.

[111] Tian, Feng, RasmusFensholt, Jan Verbesselt, Kenneth Grogan, Stephanie Horion, and Yunjia Wang. "Evaluating temporal consistency of long-term global NDVI datasets for trend analysis." Remote Sensing of Environment 163 (2015): 326-340.

[112] Oh, Kwan-Jung, Hyon-GonChoo, and Jinwoong Kim. "Temporal Consistency Enhancement for Digital Holographic Video." In Advances in Multimedia Information Processing-PCM 2015, pp. 616-622. Springer International Publishing, 2015.

[113] Schwartz, Ernst, AndrasJakab, GregorKasprian, LillaZöllei, and Georg Langs. "A Locally Linear Method for Enforcing Temporal Smoothness in Serial Image Registration." In Spatio-temporal Image Analysis for Longitudinal and Time-Series Image Data, pp. 13-24. Springer International Publishing, 2015.

[114] Rockinger, O. (1997, October). Image sequence fusion using a shift-invariant wavelet transform. In Image Processing, 1997. Proceedings., International Conference on (Vol. 3, pp. 288-291). IEEE.

[115] Toet, A. (1992). Multiscale contrast enhancement with applications to image fusion. Optical Engineering, 31(5), 10261031 . 